\title{
A survey assessing adoption of the ACMG-AMP guidelines for interpreting sequence variants and identification of areas for continued improvement
}

\author{
Annie Niehaus, $\mathrm{BA}^{1,2}$, Danielle R. Azzariti, $\mathrm{MS}^{2,3}$, Steven M. Harrison, $\mathrm{PhD}^{2,3}$, \\ Marina T. DiStefano, $\mathrm{PhD}^{2}$, Sarah E. Hemphill, BA ${ }^{2}$, Ozlem Senol-Cosar, $\mathrm{PhD}^{2}$ and \\ Heidi L. Rehm, PhD $2,3,4$
}

Genetics in Medicine (2019) 21:1699-1701; https://doi.org/10.1038/s41436-018-0432-7

In March 2015, a workgroup of the American College of Medical Genetics and Genomics (ACMG) and the Association of Molecular Pathology (AMP) published standards for the Interpretation of Sequence Variants. ${ }^{1}$ These guidelines recommended a systematic scoring process for analyzing evidence and classifying variants into five tiers. In addition, they recommended the following terminology for sequence variant interpretation in Mendelian genes: pathogenic (P), likely pathogenic (LP), uncertain significance (VUS), likely benign (LB), and benign (B). If pathogenicity is asserted, these guidelines advised laboratories to specify condition and mode of inheritance.

While these guidelines have been widely referenced by clinical laboratories, the adoption of these guidelines and how laboratories are incorporating them into variant interpretation workflows has not been assessed. To evaluate the utilization of the ACMG-AMP terminology and scoring process, the National Institutes of Health (NIH)-funded Clinical Genome Resource surveyed clinical testing laboratories registered in the Genetic Testing Registry (GTR; https:// www.ncbi.nlm.nih.gov/gtr/). ${ }^{2}$ In July 2017, an invitation email was sent to one representative from each of the 195 US-based and 170 international laboratories. The survey assessed whether or not a laboratory had adopted the terminology and scoring system recommended in the ACMG-AMP guidelines, as well as reasons why or why not. Representatives from 65 laboratories responded, including individuals from 33 international laboratories representing 15 different countries. Respondents from Canada $(n=9)$, the United Kingdom $(n=$ $4)$, and Australia $(n=4)$ accounted for the highest representation among international laboratories.

Analysis of survey responses demonstrated high adoption of the ACMG-AMP terminology recommendations. Ninety-five percent of surveyed laboratories (62/65 respondents) reported using the ACMG-AMP five tiers for classifying variants in Mendelian genes. International laboratories were just as likely to report using the guidelines as US-based laboratories. Eighty-six percent of surveyed laboratories classified variants with respect to a condition and mode of inheritance in a uniform and structured manner, and $89 \%$ of laboratories routinely avoided the use of "mutation" or "polymorphism" in clinical reports in favor of "variant."

The ACMG-AMP guidelines recommended that laboratories use at least the five classification tiers (P, LP, VUS, LB, B), but allowed laboratories to optionally use more tiers. While $78 \%$ $(51 / 65)$ of responding laboratories use just the five classification tiers, $22 \%$ of laboratories $(14 / 65)$ indicated that they use additional terms, such as subcategories (e.g., VUS-favor benign), to further classify variants. Examples of these subcategories provided by respondents included "uncertain clinical significance, possibly pathogenic" and "topline VUS." It should be noted that this survey did not delineate between the use of subcategories within a laboratory's internal databases versus what is reported or submitted to ClinVar, which currently does not accept these subcategories (ClinVar: https:// www.ncbi.nlm.nih.gov/clinvar/).

In regard to the evidence criteria provided in the ACMGAMP guidelines (Figure 1 of ref. ${ }^{1}$ ), 36\% of laboratories $(n=$ 22 ) use the evidence criteria exactly as described, $44 \%$ ( $n=$ $28)$ use an approach that is roughly consistent, and $17 \%(n=$ 11) use an approach that they considered more advanced than the ACMG-AMP approach, for a total of $97 \%$ of laboratories (62/64) using approaches that they considered consistent with these professional guidelines. The fact that many laboratories have felt the need to modify or evolve the criteria is consistent with reports that the criteria may be inconsistently applied

\footnotetext{
${ }^{1}$ College of Medicine, Medical University of South Carolina, Charleston, SC, USA; ${ }^{2}$ Laboratory for Molecular Medicine, Partners Healthcare, Cambridge, MA, USA; ${ }^{3}$ Medical and Population Genetics, Broad Institute of MIT and Harvard, Cambridge, MA, USA; ${ }^{4}$ Center for Genomic Medicine, Massachusetts General Hospital, Boston, MA, USA. Correspondence: Heidi L. Rehm (hrehm@broadinstitute.org)
}

Submitted 9 October 2018; accepted: 21 December 2018

Published online: 23 January 2019 
across groups. $^{3}$ As an example of groups evolving the guideline, Nykamp and colleagues reported implementing a refined version of the guideline to create more consistency and accuracy in the application of the guideline. ${ }^{4}$ Jarvik and Browning proposed additional guidance for quantifying segregation, and Walsh and colleagues provided guidance on the use of evidence from tumors. ${ }^{5,6}$ In addition, ClinGen's Sequence Variant Interpretation Working Group has published several papers and posted additional guidance around many criteria, with more under development (https://www. clinicalgenome.org/working-groups/sequence-variantinterpretation/). ${ }^{7-9}$ Furthermore, ClinGen's Variant Curation Expert Panels are publishing specifications of the guidelines to further clarify when certain criteria are applicable for a given gene or disease and what thresholds and strengths of evidence are appropriate for certain criteria. ${ }^{10-15}$

This survey also identified that the community would benefit from consensus on how to annotate low penetrance variants in genes for Mendelian disorders. Forty-eight percent $(30 / 63)$ of laboratories responded that they describe such variants using the standard five-tier terminology, but add qualifiers to denote low penetrance or a mild effect (e.g., pathogenic, low penetrance). Fourteen percent of responding laboratories described such variants as "risk alleles" with a confidence level, $13 \%$ of laboratories used an alternate terminology system, and $25 \%$ of laboratories did not have a specific terminology system in place for such variants. These results are consistent with a publication by Yang and colleagues that reports that a large source of discordance in ClinVar is due to variants with low penetrance. ${ }^{16}$ In response to this area of needed guidance, ClinGen has launched a task team that is beginning to develop further recommendations for the classification of low penetrance variants.

Finally, continued efforts are needed to encourage laboratories to submit to ClinVar given the important role of data sharing in improving consistency and accuracy of variant interpretation. As of July 2017, only 11\% (7/63) of respondents regularly submitted at least $75 \%$ of their variants to ClinVar once a year. Thirty-three percent (21/63) submitted in the past but did not do so regularly or have only submitted a limited set of variants, $29 \%$ of laboratories had never submitted but hoped to start within the next year, and $27 \%$ did not submit at all. It has become clear that no matter how detailed guidelines are for variant interpretation, laboratories do not always interpret variants consistently, either due to varied application of evidence criteria or the use of alternate sources or unpublished evidence. ${ }^{3,17}$ Yet sharing within ClinVar has enabled resolution of the majority of differences assessed, emphasizing the critical importance of laboratory submission to ClinVar as a component of consistency in the application of variant interpretation guidelines. $^{9,17,18}$ To promote recognition of laboratories that engage in data sharing and interpretation discrepancy resolution efforts, ClinGen has published a list of laboratories (www. clinicalgenome.org/lablist) that meet minimum requirements for data sharing. This list can be referenced by care providers and payers to guide their decision making on where to order genetic testing and when to reimburse testing. Interested clinical testing laboratories are encouraged to contact ClinGen to participate in these important data sharing activities.

In summary, the ACMG-AMP guidelines provided an evidence-based approach for classifying and interpreting sequence variants. The survey described here demonstrated widespread adoption of the recommendations in just three years. However, the guidelines noted that further refinement and disease specifications would be needed to continue to improve the accuracy and consistency of variant interpretation and our survey has confirmed this need for ongoing development. Future efforts should focus on the refinement of criteria, development of consensus guidance for how to annotate low penetrance variants in genes for Mendelian disorders, recommendations of standard terms for subcategories of variants of uncertain significance, and methods to incentivize widespread deposition of variant interpretations into ClinVar.

\section{ACKNOWLEDGEMENTS}

We thank the laboratories for responding to our survey.

\section{FUNDING}

This study was supported by the National Human Genome Research Institute (NHGRI) under award U41HG006834. The authors received $\mathrm{NIH}$ funding in support of this work. The content is solely the responsibility of the authors and does not necessarily represent the official views of the $\mathrm{NIH}$.

\section{DISCLOSURE}

The authors declare no conflicts of interest.

Publisher's note: Springer Nature remains neutral with regard to jurisdictional claims in published maps and institutional affiliations.

\section{REFERENCES}

1. Richards S, Aziz N, Bale S, et al. Standards and guidelines for the interpretation of sequence variants: a joint consensus recommendation of the American College of Medical Genetics and Genomics and the Association for Molecular Pathology. Genet Med. 2015;17:405.

2. Rehm HL, Berg JS, Brooks LD, et al. ClinGen-the clinical genome resource. N Engl J Med. 2015;372:2235-2242.

3. Amendola LM, Dorschner MO, Robertson RD. et al. Actionable exomic incidental findings in 6503 participants: challenges of variant classification. Genome Res. 2015;25:305-315.

4. Nykamp K, Anderson M, Powers M, et al. Sherloc: a comprehensive refinement of the ACMG-AMP variant classification criteria. Genet Med. 2017:19:1105.

5. Jarvik GP, Browning BL. Consideration of cosegregation in the pathogenicity classification of genomic variants. Am J Hum Genet. 2016:98:1077-1081.

6. Walsh MF, Ritter DI, Kesserwan C, et al. Integrating somatic variant data and biomarkers for germline variant classification in cancer predisposition genes. Hum Mutat. 2018;39:1542-1552. 
7. Ghosh R, Harrison $\mathrm{SM}$, Rehm HL, et al. Updated recommendation for the benign stand-alone ACMG/AMP criterion. Hum Mutat. 2018, 39:1525-1530.

8. Abou Tayoun AN, Pesaran T, DiStefano MT, et al. Recommendations for interpreting the loss of function PVS1 ACMG/AMP variant criterion. Hum Mutat. 2018:39:1517-1524.

9. Harrison SM, Dolinksy JS, Chen W, et al. Scaling resolution of variant classification differences in ClinVar between 41 clinical laboratories through an outlier approach. Hum Mutat. 2018;39:1641-1649.

10. Gelb BD, Cavé H, Dillon MW. et al. ClinGen's RASopathy Expert Panel consensus methods for variant interpretation. Genet Med. 2018:20:1334-1345.

11. Kelly MA, Caleshu C, Morales A, et al. Adaptation and validation of the ACMG/AMP variant classification framework for MYH7-associated inherited cardiomyopathies: recommendations by ClinGen's Inherited Cardiomyopathy Expert Panel. Genet Med. 2018;20:351.

12. Lee K, Krempely K, Roberts ME, et al. Specifications of the ACMG/AMP variant curation guidelines for the analysis of germline $\mathrm{CDH} 1$ sequence variants. Hum Mutat. 2018;39:1553-1568.
13. Zastrow DB, Baudet $H$, Shen $W$, et al. Unique aspects of sequence variant interpretation for inborn errors of metabolism (IEM): the ClinGen IEM Working Group and the Phenylalanine Hydroxylase Gene. Hum Mutat. 2018;39:1569-1580.

14. Mester JL, Ghosh R, Pesaran T, et al. Gene-specific criteria for PTEN variant curation: recommendations from the ClinGen PTEN Expert Panel. Hum Mutat. 2018;39:1581-1592.

15. Oza AM, DiStefano MT, Hemphill SE, et al. Expert specification of the ACMG/AMP variant interpretation guidelines for genetic hearing loss. Hum Mutat. 2018;39:1593-1613.

16. Yang $S$, Lincoln SE, Kobayashi $Y$, et al. Sources of discordance among germ-line variant classifications in ClinVar. Genet Med. 2017; 19:1118.

17. Harrison SM, Dolinsky JS, Johnson AEK, et al. Clinical laboratories collaborate to resolve differences in variant interpretations submitted to ClinVar. Genet Med. 2017;19:1096.

18. Garber KB, Vincent LM, Alexander JJ, et al. Reassessment of genomic sequence variation to harmonize interpretation for personalized medicine. Am J Hum Genet. 2016;99:1140-1149. 